\title{
Uncommon Pathogens Causing Hospital-Acquired Infections in Postoperative Cardiac Surgical Patients
}

\author{
Manoj Kumar Sahu ${ }^{1}$ Netto George ${ }^{2} \quad$ Neha Rastogi ${ }^{2}$ \\ ${ }^{1}$ Department of Cardiothoracic and Vascular Surgery, CN Centre, All \\ India Institute of Medical Sciences, Ansari Nagar, New Delhi, India \\ 2Infectious Disease, Department of Medicine, All India Institute of \\ Medical Sciences, Ansari Nagar, New Delhi, India
}

\author{
Chalatti Bipin ${ }^{1}$ Sarvesh Pal Singh ${ }^{1}$
}

J Card Crit Care 2020;3:89-96

\begin{abstract}
Address for correspondence Manoj K Sahu, MD, DNB, Department of Cardiothoracic and Vascular Surgery, CTVS office, 7th floor, CN Centre, All India Institute of Medical Sciences, New Delhi-110029, India (e-mail:drmanojsahu@gmail.com).
\end{abstract}

\begin{abstract}
Bacterial infections are common causes of sepsis in the intensive care units. However, usually a finite number of Gram-negative bacteria cause sepsis (mostly according to the hospital flora). Some organisms such as Escherichia coli, Acinetobacter baumannii, Klebsiella pneumoniae, Pseudomonas aeruginosa, and Staphylococcus aureus are relatively common. Others such as Stenotrophomonas maltophilia, Chryseobacterium indologenes, Shewanella putrefaciens, Ralstonia pickettii, Providencia, Morganella species, Nocardia, Elizabethkingia, Proteus, and Burkholderia are rare but of immense importance to public health, in view of the high mortality rates these are associated with. Being aware of these organisms, as the cause of hospital-acquired infections, helps in the prevention,

Keywords

- uncommon pathogens

- hospital-acquired infection

- cardiac surgical intensive care unit treatment, and control of sepsis in the high-risk cardiac surgical patients including in heart transplants. Therefore, a basic understanding of when to suspect these organisms is important for clinical diagnosis and initiating therapeutic options. This review discusses some rarely appearing pathogens in our intensive care unit with respect to the spectrum of infections, and various antibiotics that were effective in managing these bacteria.
\end{abstract}

\section{Introduction}

Despite utmost care and strict aseptic precautions in the pre-, intra-, and postoperative period for cardiovascular surgeries, many a times some of the patients contract hospital-acquired infections (HAIs) in the intensive care unit (ICU) culminating in sepsis. Though many hospitals have tailor-made antibiotic protocol for surgical prophylaxis and treatment in controlling such infections, certain rare organisms escape the usual empirical regime treatment and pose serious morbidity and even cause mortality in some patients.

Difficulties in isolating these uncommon pathogens range from not suspecting those as a cause of HAI, to not doing the relevant investigations pertaining to the organism. Added to this, some organisms colonize the patients, incubate, and lead to serious infections during their hospital stay or the period immediately after that. Patients with immunocompromised state (postorgan transplant patients) and chronic infections are more vulnerable to these pathogens, developing life-threatening HAIs.

During the study period from June 2017 to December 2018, we had a total number of 307 HAIs comprising of $3.63 \%$ of the total ICU admissions. The prominent pathogens causing nosocomial infections during the above time period were Acinetobacter (29.9\%), Escherichia coli (23.45\%), Klebsiella spp. (17.9\%), Pseudomonas aeruginosa (7.49\%), methicillin-resistant Staphylococcus aureus (1.3\%), coagulase-negative Staphylococcus (6.4\%), Enterococcus (3.9\%), and Enterobacter (2.9\%). Rest of the others were uncommonly occurring organisms, and overall majority of the HAIs are due to Gram-negative bacteria.

Below mentioned are few such organisms that were isolated from patients in the cardiac surgical ICU of an apex care institute from June 2017 to December 2018. A knowledge of these bacteria will help in suspecting such published online

March 6, 2020
DOI https://doi.org/

10.1055/s-0040-1705080 ISSN 2457-0206.
(C2020 Official Publication of

The Simulation Society (TSS), accredited by International Society of Cardiovascular Ultrasound (ISCU).
License terms

() (1) $\ominus \circledast$ 
infections, when patients are not improving on regular protocolized antibiotic coverage.

\section{Stenotrophomonas maltophilia}

\section{Case Scenario}

Four of our patients (two children and two adults: 4/307; $1.3 \%)$ were infected with Stenotrophomonas, which was isolated from blood or endotracheal aspirate. These bacteria were sensitive to cotrimoxazole, fluroquinolones, and chloramphenicol. Two of our patients (50\%) succumbed to the sepsis despite the antibiotic escalation based on reports.

\section{Description}

Stenotrophomonas maltophilia, earlier classified as Pseudomonas or Xanthomonas maltophilia, ${ }^{1}$ is an aerobic, nonglucose-fermenting, nonspore-forming, nonacid-fast facultative Gram-negative rod. It is emerging as a multidrug-resistant (MDR) global opportunistic pathogen. It survives on almost any humid surface and has been isolated from a wide variety of aquatic sources, such as suction tubing, nebulizers, and endoscopes. ${ }^{2}$

\section{Epidemiology}

Stenotrophomonas maltophilia is a major nosocomial bacterial pathogen as is P. aeruginosa. ${ }^{3}$ It has a propensity to grow in patients with weakened immune system, especially in neutropenic patients. It is not extremely uncommon as a cause of HAI, mostly causes respiratory tract infections (pneumonia), and is associated with high mortality (23-44\%) as stated by Gokhan Gozel et al. ${ }^{4}$ Patients at increased risk of acquiring HAIs with Stenotrophomonas are those having recent history of antibiotic therapy, human immunodeficiency virus (HIV) infection, malignancies, or corticosteroid or immunosuppressant therapy. ${ }^{2}$

\section{Organs Affected}

Infections involving lungs (pneumonia) are the commonest, followed by bloodstream, urinary tract, skin, soft tissue, bone and joint, heart, meninges, and eyes. ${ }^{2}$

\section{Treatment}

Infections caused by Stenotrophomonas are difficult to treat because of their ability to form biofilm and produce various extracellular enzymes. They are intrinsically resistant to a variety of antibiotics including penicillin, cephalosporins, carbapenems, and aminoglycosides. ${ }^{2}$ Limited therapeutic options exist; the treatment of choice is usually trimethoprim-sulfamethoxazole. $^{5}$ Other potential alternatives include ceftazidime, chloramphenicol, levofloxacin, minocycline, and ticarcillin-clavulanate, as has been depicted in various studies. ${ }^{6}$ Combination therapy is warranted in life-threatening infections including bacteremia and pneumonia. ${ }^{3,7,8}$

\section{Chryseobacterium indologenes}

\section{Case Scenario}

Six of our patients developed sepsis caused by Chryseobacterium spp. (6/307; $1.9 \%)$, yielded from respiratory secretions and blood. Among them, three were in the extreme age group (one neonate, one infant, and a 94-year-old), where the organism was resistant to almost all the antibiotics tested. Two of them succumbed to the illness; the neonate responded to levofloxacin and survived. Among the other three survivors, the cultures showed sensitivity to tigecycline, cotrimoxazole, ciprofloxacin, and piperacillin-tazobactam.

\section{Description}

Chryseobacterium indologenes, formerly known as Flavobacterium indologenes, are Gram-negative, aerobic, nonfermentative, oxidase, and catalase-positive nonmotile bacilli. Six species of Chryseobacterium are commonly isolated from clinical specimens: C. meningosepticum, C. odoratum, C. multivorum, C. breve, C. indologenes, and C. gleum. ${ }^{9}$

\section{Epidemiology}

Nosocomial infections due to $C$. indologenes have been linked to the use of distilled water/intravenous fluids in equipment such as respirators, mist tents, humidifiers, incubators, ice chests, and syringes. ${ }^{10}$ It resists chlorination and can survive in municipal water supplies. ${ }^{11}$ The bacterium has ability to form biofilms, ${ }^{12}$ which make it difficult to treat, particularly in cases of indwelling device-related infections, despite being considered as a low-virulence organism. ${ }^{11,13} \mathrm{~A}$ 5-year SENTRY program has reported $C$. indologenes to be a significant nosocomial pathogen. ${ }^{12}$ Most cases have been reported in immunocompromised patients and in those suffering from malignancies.

\section{Organs Affected}

Reported infections include bacteremia, urinary tract infection (UTI), ventilator-associated pneumonia, pyonephritis, biliary tract infection, peritonitis, ${ }^{14}$ ocular infections, and surgical and burn wound infections. ${ }^{13}$ Infections are seen in patients with long-term indwelling devices like chest drains, peritoneal catheters, central venous catheters (CVC), tracheostomy tubes, and urinary catheters, and in those having prolonged exposure to broad-spectrum antibiotics.

\section{Treatment}

Appropriate choice of antibiotics for the treatment of Chryseobacterium infection is difficult because of the unpredictability of antimicrobial resistance of this organism, which often shows resistance to many of the antibiotics chosen empirically for serious Gram-negative infections.

Chryseobacterium species exhibit MDR due to production of class A and class B lactamases and are susceptible to quinolones. ${ }^{12}$ 
Chryseobacterium has intrinsic resistance to aminoglycosides, first-generation cephalosporins, aminopenicillins, carbapenems, and aztreonam. ${ }^{15}$ The most active agents against Chryseobacterium are quinolones (gatifloxacin and levofloxacin) and trimethoprim-sulfamethoxazole with $>95 \%$ susceptibility. ${ }^{16}$

\section{Shewanella putrefaciens}

\section{Case Scenario}

The patient was a 7 -month-old infant $(1 / 307 ; 0.3 \%)$ who underwent postcardiac surgery; this organism was isolated from respiratory secretion and was sensitive to netilmicin and colistin. He responded well to the appropriate antibiotic escalation.

\section{Description}

Shewanella putrefaciens was first isolated in 1931 from putrefied butter and was successively called Achromobacter putrefaciens, Pseudomonas putrefaciens, Alteromonas putrefaciens, and, finally, Shewanella spp. ${ }^{17}$ It currently belongs to the genus Shewanella, which also includes Shewanella algae. ${ }^{18}$ It is a Gram-negative pleomorphic bacterium, facultative anaerobe, part of marine microflora, and very rarely becomes pathogenic to humans.

\section{Epidemiology}

The only species that have been isolated from clinical specimens are S. putrefaciens and S. algae. ${ }^{17}$ Shewanella spp. have in rare cases been found to be associated with medical devices leading to HAI outbreaks. ${ }^{17}$ In many cases, it was isolated in polymicrobial infections.

Few cases were found to be colonizers. Maximum cases were reported from Denmark, South Korea, and South Africa, and $11 \%$ of the total reported cases were in immunocompromised patients. A skin or mucosal portal of entry was found for $53 \%$ of the patients and exposure to the marine environment was reported for $44 \%$. Also, $28 \%$ of cases showed bacteremia and the mortality rate was $13 \%$. Shewanella algae appears to be more closely related to human illnesses than S. putrefaciens. ${ }^{18}$

\section{Organs Affected}

Skin and soft tissue injuries (33\%) were the most reported portal of infection ${ }^{19}$ and cellulitis was the most common clinical manifestation. Other manifestations were ear infections, abscesses, fasciitis, myonecrosis, pneumonia, nonhealing ulcers, and peritonitis. However, $28 \%$ immunocompromised patients may develop bacteremia. ${ }^{17,19}$

\section{Treatment}

Most Shewanella species respond in vitro to a broad range of antibiotics, such as third- and fourth-generation cephalosporins, carbapenems, aminoglycosides, and fluoroquinolones. These can show resistance to imipenem by secreting an oxacillinase. Most isolates were susceptible to cefotaxime (95\%), piperacillin-tazobactam (98\%), gentamicin (99\%), and ciprofloxacin (94\%). ${ }^{17}$ Less than $25 \%$ susceptibility was noted to penicillin and none of the isolates were susceptible to fosfomycin, whereas only 53\% were susceptible to colistin.

\section{Ralstonia pickettii}

\section{Case Scenario}

A 94-year-old patient had Ralstonia pickettii (1/307; 0.3\%) isolated from blood cultures sensitive to amikacin, cephalosporins, piperacillin-tazobactam, and carbapenems. Despite appropriate antibiotic escalation, the patient succumbed to the illness.

\section{Description}

Ralstonia pickettii and Ralstonia insidiosa are waterborne bacteria. They survive, grow in various water sources, and are emerging pathogens in hospital settings. They are Gram-negative, aerobic, nonfermentative rod-shaped, lowvirulence organisms. ${ }^{20}$

\section{Epidemiology}

First isolated in $1973,{ }^{21}$ it was initially considered belonging to Pseudomonas and later was reclassified under Burkholderia pickettii; however, in 1995 it was named Ralstonia. ${ }^{22}$ Eleven species ${ }^{21}$ of Ralstonia have been described of which R. pickettii is considered as a potential etiology of HAI among patients who are immunocompromised, suffering from cystic fibrosis, and having CVC. ${ }^{23}$

\section{Organs Affected}

Many cases of Ralstonia spp. are reported due to the use of contaminated solutions, sterile water, hand washes, dialysis fluid, as well as via the colonization of indwelling devices. ${ }^{20}$ Ralstonia pickettii has been identified in cases of primary bacteremia, pneumonia, infective endocarditis, peritonitis, and meningitis. ${ }^{20}$

\section{Treatment}

There are no treatment guidelines for Ralstonia bacteremia owing to the scarcity of volume of cases. However, sine inducible $\beta$-lactamases blaOXA- 60 and blaOXA- 22 are present in this bacterium, it is considered responsible for the high level of resistance to $\beta$-lactams. Often, it is considered resistant to aminoglycosides. ${ }^{20}$ Natural resistance of this organism to polymyxins is well known. ${ }^{24}$

However, this bacterium has been found to be susceptible to third-generation cephalosporins, quinolones, and carbapenems. ${ }^{25}$ In a review of 11 cases by Mikulska et al, Ralstonia was susceptible to aminoglycosides, fluoroquinolones, thirdand fourth-generation cephalosporins, piperacillin-tazobactam, and carbapenems, while also resistant to aztreonam. ${ }^{26}$

\section{Providencia Species}

\section{Case Scenario}

A 4-year-old girl had urosepsis due to Providencia rettgeri $(1 / 307 ; 0.3 \%)$ in the postoperative period. The cultures 
showed sensitivity to cephalosporins and tigecycline. Despite appropriate antibiotics, patient did not recover.

\section{Description}

Providencia is a genus within the Enterobacteriaceae family. There are currently eight recognized species within the genus: P. alcalifaciens, $P$. burhodogranaeriae, $P$. heimbachae, $P$. rettgeri, $P$. rustigianii, $P$. sneebia, $P$. stuartii, and P. vermicola. ${ }^{27}$ Providencia stuartii is the most frequently encountered human pathogen within the genus.

\section{Epidemiology}

The most frequent site of isolation is the urinary tract of chronically catheterized patients in hospitals and long-term care facilities. ${ }^{27}$ However, it has also been reported in many cases of travelers' diarrhea. A report from Nepal in 2014, mentioning a cluster of surgical infections with P. rettgeri, demonstrated the presence and significance of this organism in the Asia-Pacific region. ${ }^{28}$ While Providencia species do not routinely cause UTI or bacteremia, the overall mortality rate from bacteremia is high in the elderly with severe underlying conditions. ${ }^{27}$

\section{Organs Affected}

Besides UTI, wound infections and pneumonia may also occur, particularly in critically ill patients. Providencia rettgeri has been implicated as a causative agent of "purple bag syndrome," where the enzymatic activity deaminate aromatic amino acids like tryptophan and phenylalanine and influence the formation of indole and indoxyl sulfate, which are metabolites of tryptophan thus causing purple-colored urine..$^{29}$

\section{Treatment}

Providencia rettgeri strains are intrinsically resistant to many antimicrobials including ampicillin, first-generation cephalosporins, polymyxins, and tigecycline. ${ }^{30}$ It is typically resistant to gentamicin and tobramycin but susceptible to amikacin. ${ }^{29}$ The New Delhi metallo- $\beta$-lactamase- 1 gene facilitates the production of an enzyme called carbapenemase by Providencia species, which makes the bacteria resistant to carbapenems. Providencia sp. was identified as one of the most common MDR pathogens responsible for UTI.

\section{Morganella morganii}

\section{Case Scenario}

We encountered one 3-month-old baby postcardiotomy with Morganella sepsis (1/307; 0.3\%). The isolate was sensitive to amikacin, carbapenems, piperacillin-tazobactam, and cephalosporins. However, the child succumbed to the illness despite antibiotic escalation.

\section{Description}

Morganella morganii belongs to the tribe Proteeae of family Enterobacteriaceae. It is a facultative Gram-negative, anaerobic rod. ${ }^{31}$

\section{Epidemiology}

Morganella species is found in the environment and in the intestinal tracts of humans, mammals, and reptiles as part of the normal flora. This rarely causes infections in healthy individuals, but causes HAIs in diabetics, neonates, and postoperative patients. It was originally thought to be a cause of summer diarrhea. ${ }^{32}$ This bacterium was first found to be a cause of UTI in $1939 .{ }^{33}$ In 1983, some bacteremia cases due to the organism was reported in a cardiac surgery unit. ${ }^{33}$

Morganella morganii bacteremia is rare and accounts for $0.69 \%$ of bacteremia in adults as reported by Williams et $\mathrm{al}^{34}$ but a higher incidence in newborns at 3.6\% as reported by Custović and Hadzić. ${ }^{35}$ Bacteremia caused by M. morganii infection can be associated with a high mortality rate of 22 to $38 \%{ }^{31}$

\section{Organs Affected}

Morganella sepsis usually follows skin, soft tissue, and urinary or biliary tract infection. ${ }^{36}$ Urinary tract is the most common infection site. Most often it occurs in elderly patients in nursing homes with long-term indwelling catheters. ${ }^{37}$ Morganella also causes intra-abdominal infection. In a retrospective review of 61 cases of M. morganii bacteremia, Kim found that $64 \%$ were related to intra-abdominal infections (including biliary infection, liver abscess, and peritonitis). ${ }^{38}$

\section{Treatment}

Morganella strains are naturally resistant to penicillin, ampicillin, ampicillin/sulbactam, oxacillin, first- and secondgeneration cephalosporins, erythromycin, tigecycline, colistin, and polymyxin B. ${ }^{32,33}$ Most strains are susceptible to piperacillin, third- and fourth-generation cephalosporins, carbapenems, aztreonam, fluoroquinolones, aminoglycosides, and chloramphenicol. ${ }^{33}$

Morganella species are capable of producing inducible chromosomal Amp C $\beta$-lactamases rendering them resistant to action of primary- and extended-spectrum penicillins and cephalosporins..$^{36,39}$ A third-generation cephalosporin has been suggested as the drug of choice for Morganella infections. Addition of an aminoglycoside to a cephalosporin may decrease the potential resistance to broad-spectrum cephalosporins. ${ }^{33}$ Adjunctive management like replacing urinary catheter in UTIs, or intravenous lines in case of bacteremia, is to be done. Strict infection control practices are to be followed if an organism is isolated.

\section{Nocardiosis}

\section{Case Scenario}

We had a male orthotopic heart transplant recipient (1/307; $0.3 \%$ ), who presented with progressive breathlessness and was diagnosed to have necrotizing bilateral pneumonia due to Nocardia. The patient succumbed to the illness within few days in spite of best efforts.

\section{Description}

Nocardia species are ubiquitous filamentous, branching, aerobic Gram-positive, weak acid-fast bacilli. Nocardia 
asteroides is the predominant human pathogen followed by Nocardia brasiliensis, Nocardia farcinica, and Nocardia nova. ${ }^{40}$

\section{Epidemiology}

Nocardia infections usually occur in situations of cellular immunodeficiency, such as in solid organ or bone marrow transplant recipients, ${ }^{41}$ HIV patients, and long-term users of corticosteroids and immunosuppressants (especially calcineurin inhibitors). Inhalation is the primary route of entry, but infection can also occur after direct cutaneous inoculation. ${ }^{42}$ Disseminated forms of nocardiosis have been described in immunodeficient hosts while immunocompetent hosts usually present with cutaneous infections. ${ }^{43}$ There have been several cases of endocarditis attributed to Nocardia species. Nocardia infection classically occurs in immunocompromised patients.

\section{Organs Affected}

Pulmonary infection is most common, accounting for almost $82 \%$ of the cases as reported from Australia, ${ }^{42}$ but hematogenous dissemination to other organs is possible, as there are cases of brain abscesses, ${ }^{44}$ aortitis, ${ }^{45}$ and endophthalmitis, ${ }^{46}$ following Nocardia infection. Clinical and radiological manifestations are just like tuberculosis or malignancy, which include pneumonia and infiltrative or nodular lesions, which may be accompanied by cavitation, abscess formation, pleural effusions, or empyema and sometimes mass-like lesions. ${ }^{47}$ The major differentials in diagnosis are pneumonia, tuberculosis, bronchogenic carcinoma, or lung abscess. Immunocompromised patients are significantly more likely to experience dissemination, bloodstream infection, prolonged hospitalization, and death. Heart transplant recipients initially had an especially high rate of Nocardia infection. In a study by Lebeaux et al, 1-year mortality was 10-fold higher in patients with nocardiosis $(16.2 \% ; 19 / 117)$ than in control transplant recipients $(1.3 \% ; 3 / 233 ; p<0.001){ }^{44}$ If the disease is not diagnosed in early stage, it disseminates to multiple organs such as brain, lymphoreticular system, or musculoskeletal system leading to high mortality of about 70 to $80 \%{ }^{47}$

\section{Treatment}

Standard therapy of Nocardia infections is a prolonged course of trimethoprim-sulfamethoxazole(52). Other treatment options in cases of drug intolerance include amikacin, imipenem, third-generation cephalosporins, minocycline, and amoxicillin-clavulanic acid, or, recently, linezolid. ${ }^{47}$

\section{Elizabethkingia meningoseptica}

\section{Case Scenario}

A 2-month-old male and a 28-year-old female with postcardiac surgery had isolates of Elizabethkingia meningoseptica from blood culture $(2 / 307 ; 0.6 \%)$. Both of them were treated as per culture sensitivity and had a favorable outcome. Another 80-year-male patient's blood culture yielded this organism, which was an MDR and did not respond to treatment.

\section{Description}

Elizabethkingia meningoseptica, an emerging hospital-acquired pathogen, was previously known as C. meningosepticum until 2005 and Flavobacterium meningosepticum until 1994.48,49 It is a ubiquitous Gram-negative, aerobic, nonfermentative, nonmotile, nonspore-forming bacteria. ${ }^{48}$

\section{Epidemiology}

Elizabethkingia spp. are frequently found in soil, fresh and salt water, hospital water supplies, disinfectants, and medical devices. Elderly, newborns, and immunocompromised patients are most vulnerable to Elizabethkingia infection..$^{50}$ Epidemics have been reported in neonatal ICUs. ${ }^{51}$ Immunosuppression, underlying medical diseases, malignancies, prolonged hospital stay, prior use of higher antibiotics, and indwelling invasive devices like $\mathrm{CVC}^{52}$ are some of the risk factors associated with acquisition of this infection. At one medical center in northern Taiwan, a large case series showed that the yearly incidence of Elizabethkingia bacteremia increased substantially since 2002 (from 6.8-13.1 to $26.6-39.9$ per 100,000 admissions)..$^{53}$ The potential for this bacteria to display a strong biofilm biotype might also explain the failure of many of the infection control interventions during outbreaks. ${ }^{54}$ Mortality associated with Elizabethkingia infection is alarmingly high (33-52\%).

\section{Organs Affected}

Neonatal meningitis is the most common disease caused by this organism; however, pneumonia is more common in adults. Prematurity is a primary risk factor for Elizabethkingia infection. ${ }^{12}$ It has been implicated in bacteremia, meningitis, endocarditis, cellulitis, abdominal infection, wound infection, sinusitis, epididymitis, dialysis-associated peritonitis, septic arthritis, eye infections, and community acquired necrotizing fasciitis.

\section{Treatment}

Choosing an effective antibiotic against Elizabethkingia is quite difficult because no interpretive minimum inhibitory concentration and breakpoints of antibiotics against this organism have been reported. ${ }^{55}$ Elizabethkingia spp. are resistant to multiple antibiotics, especially to aminoglycosides, $\beta$-lactam antibiotics, tetracyclines, and chloramphenicol.

The antibiotic profile of E. meningoseptica is different from other Gram-negative rods. It is often susceptible to agents generally used to treat infections caused by Gram-positive bacteria (rifampicin, clindamycin, erythromycin, trimethoprim-sulfamethoxazole, quinolones, and vancomycin)..$^{55}$ Many of the bacteria possess two different types of $\beta$-lactamases, namely class $A$ extended-spectrum $\beta$-lactamases (ESBLs) and class B metallo- $\beta$-lactamases; the latter confer resistance to carbapenems, which makes the treatment difficult.

\section{Burkholderia cepacia}

\section{Case Scenario}

Two infants, 1 - and 2 -month-old ( $2 / 307 ; 0.6 \%)$, following congenital cardiac surgeries yielded Burkholderia cepacia infection in blood and endotracheal aspirate, respectively. Both these infants were successfully treated with carbapenems. 


\section{Description}

Burkholderia cepacia is a nonfermenting, aerobic, motile, opportunistic Gram-negative bacillus that can survive in certain disinfectants. It is named $B$. cepacia complex, as it contains around 20 genomovars, including $B$. cenocepacia, $B$. multivorans, $B$. vietnamiensis, $B$. dolosa, and B. cepacia. ${ }^{56}$ The bacteria can migrate across epithelial barriers and can involve lung parenchyma and microvasculature.

\section{Epidemiology}

Burkholderia cepacia is an opportunistic pathogen that causes disease primarily among immunocompromised populations. ${ }^{57}$ It is associated with outbreaks involving infections of the bloodstream, respiratory tract, and urinary tract in ICU settings. The most common occurrence of this bacterium is in association with cases of cystic fibrosis, where it is a common colonizer of the lung. ${ }^{57}$ Outbreaks have been reported originating from diverse sources such as contaminated nebulizers, antiseptics, disinfectants, ${ }^{58}$ alcohol-free mouthwash, multidose albuterol vials used among multiple patients, tap water, bottled water, cosmetics, napkins, nasal sprays, and ultrasound gel..$^{56}$ Burkholderia cepacia can spread by person-to-person contact, contact with contaminated surfaces, and exposure to B. cepacia in the environment. Hence, infection control surveillance and hand hygiene have prime importance in controlling outbreak. A study by Abdallah et al noticed 17 outbreaks of Burkholderia in adult ICUs, of which B. cepacia was the most common genomovar. ${ }^{59}$

\section{Organs Affected}

A variety of human infections caused by B. cepacia include bacteremia, septic arthritis, UTIs, peritonitis, and respiratory tract infections. Nosocomial pneumonia was observed in ventilated patients following Burkholderia infections. Cases of skin and soft tissue infections have been reported following burns. ${ }^{60}$ As it takes prolonged time for growth in cultures, this organism is quite often missed, since the medium gets discarded before its growth. ${ }^{61}$

\section{Treatment}

Burkholderia cepacia is intrinsically resistant to aminoglycosides and polymyxins, and variably resistant to $\beta$-lactams and chloramphenicol.57,62,63 The effective drugs against B. cepacia include ceftazidime, minocycline, meropenem, and cotrimoxazole. The multiple-antibiotic resistance of $B$. cepacia has been attributed to an impermeable selective outer membrane, an efflux pump mechanism, and the production of an inducible chromosomal $\beta$-lactamase. ${ }^{64}$ The high level of intrinsic resistance in this organism coupled with the lack of newer or effective antibiotics make the treatment options very difficult.

\section{Proteus mirabilis}

\section{Case Scenario}

Two female postcardiac surgical patients aged 28 and 55 years $(2 / 307 ; 0.6 \%)$ had isolates of Proteus mirabilis from blood culture, with the primary source of infection being urinary tract. Both of them have been treated with imipenem as per sensitivity and both recovered.

\section{Description}

Proteus mirabilis is a Gram-negative motile rod with ability to form swarming growth and is commonly seen associated with UTIs including cystitis, pyelonephritis, and asymptomatic bacteriuria. ${ }^{65}$

\section{Epidemiology}

Proteus mirabilis causes HAIs mainly in the elderly patients and those with type 2 diabetes. ${ }^{66}$ It is predominantly associated with complicated UTIs but bacteremia is a rare entity. ${ }^{67}$ A study by Endimiani et al ${ }^{68}$ reported 25 cases of bloodstream infections with proteus over a period of 7.5 years, out of which 11 isolates were ESBL producers, which makes it difficult to treat. It is often isolated in catheter-associated UTIs and with anatomic abnormalities of the urinary tract. The production of urease might lead to crystallization of calcium and magnesium in urinary tract leading to obstruction, hydronephrosis, and, eventually, septicemia in urinary infections. ${ }^{67}$

\section{Organs Affected}

Proteus is commonly implicated in infections of urinary tract (1-10\% of all infections). ${ }^{69}$ In addition to UTIs, Proteus can also cause infections in the respiratory tract, eye, ear, nose, skin, throat, burns, aortic aneurysm ${ }^{70}$ and wounds, ${ }^{71}$ and has been implicated in neonatal meningoencephalitis, ${ }^{72}$ empyema, and osteomyelitis. Many of the infections occur by means of ascension of the organism from gastrointestinal tract, as has been proven by the isolation of same organism from stool specimens in many cases. ${ }^{66}$

\section{Treatment}

In view of the ability to produce ESBL, treatment of severe infections with Proteus mirabilis is mainly with carbapenems. ${ }^{67}$ In a study by Chen et al, ${ }^{67}$ all the isolates among the 124 cases were sensitive to carbapenems. The currently recommended treatment for acute uncomplicated cystitis with Proteus is a course of trimethoprim-sulfamethoxazole. If the sulfamethoxazole resistance rate is above 10 to $20 \%$, the recommended alternative antibiotic therapy may include fluroquinolones or fosfomycin. Most isolates of Proteus are intrinsically resistant to tetracyclines and nitrofurantoin.

\section{Summary}

Above mentioned are some of the uncommon but noteworthy pathogenic organisms isolated from our cardiac surgical ICU over the past one-and-a-half year duration. We have noticed that the mortality rates due to sepsis with these uncommon organisms are much higher as compared to the other common organisms (e.g., Pseudomonas, Klebsiella, and Acinetobacter), especially in those who are in the extreme age groups and in immunocompromised individuals.

Though Stenotrophomonas is not a very uncommon bacteria among these, the fact that it is resistant to colistin impairs 
the treatment arsenal, making it challenging to manage. Similarly, some others like Burkholderia, Nocardia, Ralstonia, and Elizabethkingia are also resistant to polymyxins.

Elizabethkingia is another rare pathogen causing HAI and is difficult to treat as it is mainly susceptible to most of the antimicrobials for Gram-positive bacteria (e.g., Vancomycin, Teicoplanin) and resistant to those treating Gram-negative sepsis including polymyxins.

It is difficult to isolate Nocardia in routine bacterial culture and the requirement of long duration of treatment makes its treatment difficult. Association of some of these bacteria causing HAI in immunocompromised and posttransplant patients warns us to take utmost caution while dealing with them.

\section{Conflict of Interest}

None.

\section{References}

1 Denton M, Kerr KG. Microbiological and clinical aspects of infection associated with Stenotrophomonas maltophilia. Clin Microbiol Rev 1998;11(1):57-80

2 Chang YT, Lin CY, Chen YH, Hsueh P-R. Update on infections caused by Stenotrophomonas maltophilia with particular attention to resistance mechanisms and therapeutic options. Front Microbiol 2015;6:893

3 Sumida K, Chong Y, Miyake N, et al. Risk factors associated with Stenotrophomonas maltophilia bacteremia: a matched case-control study. PLoS One 2015;10(7):e0133731

4 Gokhan Gozel M, Celik C, Elaldi N. Stenotrophomonas maltophilia infections in adults: primary bacteremia and pneumonia. Jundishapur J Microbiol 2015;8(8):e23569

5 Gales AC, Seifert H, Gur D, Castanheira M, Jones RN, Sader HS. Antimicrobial susceptibility of Acinetobacter calcoaceticus-Acinetobacter baumannii complex and Stenotrophomonas maltophilia clinical isolates: results from the SENTRY Antimicrobial Surveillance Program (1997-2016) Open Forum Infect Dis 2019;6(1, Suppl 1):S34-S46

6 Batra P, Mathur P, Misra MC. Clinical characteristics and prognostic factors of patients with Stenotrophomonas maltophilia infections. J Lab Physicians 2017;9(2):132-135

7 Çıkman A, Parlak M, Bayram Y, Güdücüoğlu H, Berktaş M. Antibiotics resistance of Stenotrophomonas maltophilia strains isolated from various clinical specimens. Afr Health Sci 2016;16(1):149-152

8 Shah MD, Coe KE, El Boghdadly Z, et al. Efficacy of combination therapy versus monotherapy in the treatment of Stenotrophomonas maltophilia pneumonia. J Antimicrob Chemother 2019;74(7):2055-2059

9 Brooke JS. Stenotrophomonas maltophilia: an emerging global opportunistic pathogen. Clin Microbiol Rev 2012;25(1):2-41

10 Srinivasan G, Muthusamy S, Raveendran V, Joseph NM, Easow JM. Unforeseeable presentation of Chryseobacterium indologenes infection in a paediatric patient. BMC Res Notes 2016;9:212

11 Kirby JT, Sader HS, Walsh TR, Jones RN. Antimicrobial susceptibility and epidemiology of a worldwide collection of Chryseobacterium spp: report from the SENTRY Antimicrobial Surveillance Program (1997-2001) J Clin Microbiol 2004;42(1):445-448

12 Mukerji R, Kakarala R, Smith SJ, Kusz HG. Chryseobacterium indologenes an emerging infection in the USA. BMJ Case Rep 2016;2016:bcr2016214486

13 Bhalla GS, Gupta S, Sarao MS, Kalra D. Chryseobacterium indologenes: case report of an emerging pathogen. J Mar Med Soc 2018;20:70-72
14 Imataki O, Uemura M. Chryseobacterium indologenes, a possible emergent organism resistant to carbapenem antimicrobials after stem cell transplantation. Clin Case Rep 2016;5(1):22-25

15 Das P, Karade S, Kaur K, Ramamurthy R, Ranjan P. Chryseobacterium Indologenes pneumonitis in an infant: a case report. J Clin Diagn Res 2017;11(6):DD07-DD08

16 Palewar MS, Mudshingkar SS, Dohe V, Bharadwaj R. Infection by multidrug-resistant Chryseobacterium indologenes in cases of obstructive uropathy: case series with short review. Med J Dr DY Patil Vidyapeeth 2017;10(4):376-380

17 Christakis GB, Perlorentzou SP, Chalkiopoulou I, Athanasiou A, Legakis NJ. Chryseobacterium indologenes non-catheterrelated bacteremia in a patient with a solid tumor. J Clin Microbiol 2005;43(4):2021-2023

18 Vignier N, Barreau M, Olive C, et al. Human infection with Shewanella putrefaciens and S. algae: report of 16 cases in Martinique and review of the literature. Am J Trop Med Hyg 2013;89(1):151-156

19 Pagani L, Lang A, Vedovelli C, Moling O, Rimenti G, Pristerà R, Mian P. Soft tissue infection and bacteremia caused by Shewanella putrefaciens. J Clin Microbiol 2003;41(5):2240-2241

20 Ranjan R, Chowdhary P. A rare case of Shewanella putrefaciens bacteremia in a patient of road traffic accident. Indian J Pathol Microbiol 2017;60(4):599-600

21 Tejera D, Limongi G, Bertullo M, Cancela M. Ralstonia pickettii bacteremia in hemodialysis patients: a report of two cases. Rev Bras Ter Intensiva 2016;28(2):195-198

22 Stelzmueller I, Biebl M, Wiesmayr S, et al. Ralstonia pickettii-innocent bystander or a potential threat? Clin Microbiol Infect 2006;12(2):99-101

23 Sharma D, Sharma P, Soni P, Gupta B. Ralstonia pickettii neonatal sepsis: a case report. BMC Res Notes 2017;10(1):28

24 Nasir N, Sayeed MA, Jamil B. Ralstonia pickettii bacteremia: an emerging infection in a tertiary care hospital setting. Cureus 2019;11(7):e5084, 10.7759/cureus.5084

25 Orme J, Rivera-Bonilla T, Loli A, Blattman NN. Native valve endocarditis due to Ralstonia pickettii: a case report and literature review. Case Rep Infect Dis 2015;2015:324675

26 Pellegrino FL, Schirmer M, Velasco E. de Faria LM, Santos KR, Moreira BM. Ralstonia pickettii bloodstream infections at a Brazilian cancer institution. Curr Microbiol 2008;56(3):219-223

27 Mikulska M, Durando P, Pia Molinari M, et al. Outbreak of Ralstonia pickettii bacteraemia in patients with haematological malignancies and haematopoietic stem cell transplant recipients. J Hosp Infect 2009;72(2):187-188

28 Wie S-H. Clinical significance of Providencia bacteremia or bacteriuria. Korean J Intern Med (Korean Assoc Intern Med) 2015;30(2):167-169

29 Washington MA, Barnhill J, Griffin JM. A case of wound infection with Providencia rettgeri and coincident gout in a patient from Guam. Hawaii J Med Public Health 2015;74(11):375-377

30 Sagar S, Narasimhaswamy N, D'Souza J. Providencia rettgeri: an emerging nosocomial uropathogen in an indwelling urinary catheterised patient. J Clin Diagn Res 2017;11(6):1-2

31 Shin S, Jeong SH, Lee H, Hong JS, Park M-J, Song W. Emergence of multidrug-resistant Providencia rettgeri isolates co-producing NDM-1 carbapenemase and PER-1 extended-spectrum $\beta$-lactamase causing a first outbreak in Korea. Ann Clin Microbiol Antimicrob 2018;17(1):20

$32 \mathrm{Kim} \mathrm{JH}, \mathrm{Cho} \mathrm{CR}, \mathrm{Um} \mathrm{TH}$, et al. Morganella morganii sepsis with massive hemolysis. J Korean Med Sci 2007;22(6):1082-1084

33 Liu H, Zhu J, Hu Q, Rao X. Morganella morganii, a non-negligent opportunistic pathogen. Int J Infect Dis 2016;50:10-17

34 Williams EW, Hawkey PM, Penner JL, Senior BW, Barton LJ. Serious nosocomial infection caused by Morganella morganii and Proteus mirabilis in a cardiac surgery unit. J Clin Microbiol 1983;18(1):5-9 
35 Custović A, Hadzić S. [Epidemiology of bacterial intrahospital infections in newborns]. Med Arh 2008;62(5-6):294-297

36 Erlanger D, Assous MV, Wiener-Well Y, Yinnon AM, Ben-Chetrit E. Clinical manifestations, risk factors and prognosis of patients with Morganella morganii sepsis. J Microbiol Immunol Infect 2019;52(3):443-448

37 Chen YT, Peng HL, Shia WC, et al. Whole-genome sequencing and identification of Morganella morganii KT pathogenicity-related genes. BMC Genomics 2012;13(Suppl 7) :S4

38 Kim B-N, Kim NJ, Kim M-N, Kim YS, Woo J-H, Ryu J. Bacteraemia due to tribe Proteeae: a review of 132 cases during a decade (1991-2000) Scand J Infect Dis 2003;35(2):98-103

39 Singla N, Kaistha N, Gulati N, Chander J. Morganella morganii could be an important intensive care unit pathogen. Indian J Crit Care Med 2010;14(3):154-155

40 Paramythiotou E, Papadomichelakis E, Vrioni G, et al. A life-threatening case of disseminated nocardiosis due to Nocardia brasiliensis. Indian J Crit Care Med 2012;16(4):234-237

41 Koerner MM, El-Banayosy A, Schulz U, et al. Nocardiosis in heart transplant recipients. Heart Surg Forum 2015;18(6):E250-E252

42 McGuinness SL, Whiting SE, Baird R, et al. Nocardiosis in the tropical northern territory of Australia, 1997-2014. Open Forum Infect Dis 2016;3(4):ofw208

43 Threlkeld SC, Hooper DC. Update on management of patients with Nocardia infection. Curr Clin Top Infect Dis 1997;17:1-23

44 Lebeaux D, Freund R, van Delden C, et al; European Study Group for Nocardia in Solid Organ TransplantationEuropean Study Group for Nocardia in Solid Organ Transplantation. Outcome and treatment of nocardiosis after solid organ transplantation: new insights from a European study. Clin Infect Dis 2017;64(10):1396-1405

45 Liff DA, Kraft C, Pohlel K, et al. Nocardia nova aortitis after coronary artery bypass surgery. J Am Soc Echocardiogr 2007;20(5):537.e7-537.e8

46 Sharma D, Mathur U, Gour A, Acharya M, Gupta N, Sapra N. Nocardia infection following intraocular surgery: report of seven cases from a tertiary eye hospital. Indian J Ophthalmol 2017;65(5):371-375

47 Singh AK, Shukla A, Bajwa R, Agrawal R, Srivastwa N. Pulmonary nocardiosis: unusual presentation in intensive care unit. Indian J Crit Care Med 2018;22(2):125-127

48 Arbune M, Fotea S, Nechita A, Stefanescu V. Emerging infection with Elizabethkingia meningoseptica in neonate. A case report. J Crit Care Med (Targu Mures) 2018;4(3):96-100

49 Sarma S, Kumar N, Jha A, Baveja U, Sharma S. Elizabethkingia meningosepticum: an emerging cause of septicemia in critically ill patients. J Lab Physicians 2011;3(1):62-63

50 Decker BK, Palmore TN. The role of water in healthcare-associated infections. Curr Opin Infect Dis 2013;26(4):345-351

51 Tai I-C, Liu T-P, Chen Y-J, Lien R-I, Lee C-Y, Huang Y-C. Outbreak of Elizabethkingia meningoseptica sepsis with meningitis in a well-baby nursery. J Hosp Infect 2017;96(2):168-171

52 Chawla K, Gopinathan A, Varma M, Mukhopadhyay C. Elizabethkingia meningoseptica outbreak in intensive care unit. J Glob Infect Dis 2015;7(1):43-44

53 Jean SS, Lee WS, Chen FL, Ou TY, Hsueh PR. Elizabethkingia meningoseptica: an important emerging pathogen causing healthcare-associated infections. J Hosp Infect 2014;86(4):244-249

54 Moore LS, Owens DS, Jepson A, et al. Waterborne Elizabethkingia meningoseptica in adult critical care. Emerg Infect Dis 2016;22(1):9-17
55 Joo HD, Ann SY, Ryou SH, Kim YS, Kim JW, Kim DH. Experience with Elizabethkingia meningoseptica infection in adult patients at a tertiary hospital. Korean J Crit Care Med 2015;30(4):241-248

56 Gautam V, Singhal L, Ray P. Burkholderia cepacia complex: beyond pseudomonas and acinetobacter. Indian J Med Microbiol 2011;29(1):4-12

57 Matthaiou DK, Chasou E, Atmatzidis S, Tsolkas P. A case of bacteremia due to Burkholderia cepacia in a patient without cystic fibrosis. Respir Med CME 2011;4(3):144-145

58 Antony B, Cherian EV, Boloor R, Shenoy KV. A sporadic outbreak of Burkholderia cepacia complex bacteremia in pediatric intensive care unit of a tertiary care hospital in coastal Karnataka, South India. Indian J Pathol Microbiol 2016;59(2):197-199

59 Abdallah M, Abdallah HA, Memish ZA. Burkholderia cepacia complex outbreaks among non-cystic fibrosis patients in the intensive care units: a review of adult and pediatric literature. Infez Med 2018;26(4):299-307

60 Singhal T, Shah S, Naik R. Outbreak of Burkholderia cepacia complex bacteremia in a chemotherapy day care unit due to intrinsic contamination of an antiemetic drug. Indian J Med Microbiol 2015;33(1):117-119

61 Huang CH, Jang TN, Liu CY, Fung CP, Yu KW, Wong WW. Characteristics of patients with Burkholderia cepacia bacteremia. J Microbiol Immunol Infect 2001;34(3):215-219

62 Ranjan R, Chowdhary P, Kamra A. Community acquired Burkholderia cepacia bacteraemia presenting as MODS in an immunocompetent individual: an unusual case. J Clin Diagn Res 2017;11(3):DD01-DD02

63 Rhodes KA, Schweizer HP. Antibiotic resistance in Burkholderia species. Drug Resist Updat 2016;28:82-90

64 El Chakhtoura NG, Saade E, Wilson BM, Perez F, Papp-Wallace KM, Bonomo RAA. A 17-year nationwide study of Burkholderia cepacia complex bloodstream infections among patients in the United States Veterans Health Administration. Clin Infect Dis 2017;65(8):1253-1259

65 Abrams HL. Septicemia due to Proteus vulgaris; review of the literature and report of a case cured by streptomycin. N Engl J Med 1948;238(6):185-187

66 Schaffer JN, Pearson MM. Proteus mirabilis and urinary tract infections. Microbiol Spectr 2015;3(5):10

67 Chen CY, Chen YH, Lu PL, Lin WR, Chen TC, Lin CY. Proteus mirabilis urinary tract infection and bacteremia: risk factors, clinical presentation, and outcomes. J Microbiol Immunol Infect 2012;45(3):228-236

68 Endimiani A, Luzzaro F, Brigante G, et al. Proteus mirabilis bloodstream infections: risk factors and treatment outcome related to the expression of extended-spectrum $\beta$-lactamases. Antimicrob Agents Chemother 2005;49(7):2598-2605

69 O'Hara CM, Brenner FW, Miller JM. Classification, identification, and clinical significance of Proteus, Providencia, and Morganella. Clin Microbiol Rev 2000;13(4):534-546

70 Nagiah SK, Badbess R. Unexpected source of Proteus mirabilis bacteraemia. BMJ Case Reports 2018;11(1):ii: bcr-2018-226744

71 Wang JT, Chen PC, Chang SC, et al; TSAR Hospitals. Antimicrobial susceptibilities of Proteus mirabilis: a longitudinal nationwide study from the Taiwan Surveillance of Antimicrobial Resistance (TSAR) program. BMC Infect Dis 2014; 14(1):486

72 Omoruyi EA, Evangelista M. Proteus mirabilis septicemia and meningitis in a neonate. J Med Cases 2014;5(4):245-247 